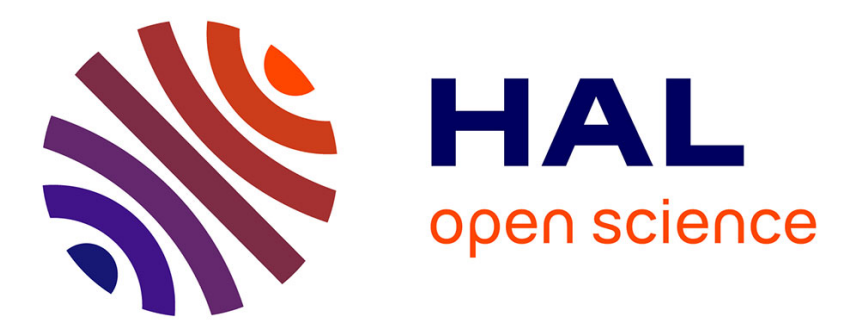

\title{
CEP-stable high-energy ytterbium-doped fiber amplifier
}

M. Natile, A. Golinelli, L. Lavenu, F. Guichard, Marc Hanna, Y. Zaouter, R. Chiche, X. Chen, J. F Hergott, W. Boutu, et al.

\section{To cite this version:}

M. Natile, A. Golinelli, L. Lavenu, F. Guichard, Marc Hanna, et al.. CEP-stable high-energy ytterbium-doped fiber amplifier. Optics Letters, 2019, 44 (16), pp.3909. 10.1364/OL.44.003909 . hal-02321849

\section{HAL Id: hal-02321849 \\ https://hal.science/hal-02321849}

Submitted on 22 Oct 2019

HAL is a multi-disciplinary open access archive for the deposit and dissemination of scientific research documents, whether they are published or not. The documents may come from teaching and research institutions in France or abroad, or from public or private research centers.
L'archive ouverte pluridisciplinaire HAL, est destinée au dépôt et à la diffusion de documents scientifiques de niveau recherche, publiés ou non, émanant des établissements d'enseignement et de recherche français ou étrangers, des laboratoires publics ou privés. 


\title{
CEP-stable high-energy ytterbium-doped fiber amplifier
}

\author{
M. Natile, ${ }^{1,2, *}$ A. Golinelli, ${ }^{3}$ L. Lavenu,${ }^{1,3}$ F. Guichard, ${ }^{1}$ M. Hanna, ${ }^{3}$ Y. Zaouter, ${ }^{1}$ R. Chiche, ${ }^{4}$ \\ X. Chen, ${ }^{1}$ J. F. Hergott, ${ }^{2}$ W. Boutu, ${ }^{2}$ (i) H. Merdjl, ${ }^{2}$ and P. Georges ${ }^{3}$ \\ ${ }^{1}$ Amplitude Laser, 2-4 rue du Bois Chaland CE 2926, 91029 Evry, France \\ ${ }^{2}$ LIDYL, CEA, CNRS, Université Paris-Saclay, UMR 9222 CEA-SACLAY, 91191 Gif-sur-Yvette, France \\ ${ }^{3}$ Laboratoire Charles Fabry, Institut d'Optique Graduate School, CNRS, Université Paris-Saclay, 91127 Palaiseau Cedex, France \\ ${ }^{4}$ Laboratoire de l'Accélérateur Linéaire, IN2P3, CNRS, Université Paris-Saclay, 91898 Orsay Cedex, France \\ *Corresponding author: michele.natile@amplitude-laser.com
}

Received 10 April 2019; accepted 10 June 2019; posted 18 June 2019 (Doc. ID 364621); published 5 August 2019

\begin{abstract}
We report on the carrier-envelope phase (CEP) stabilization of a Yb-doped fiber amplifier system delivering $30 \mu \mathrm{J}$ pulses at $100 \mathrm{kHz}$ repetition rate. A single-shot, every-shot measurement of the CEP stability based on a simple f-2f interferometer is performed, yielding a CEP standard deviation of $320 \mathrm{mrad} \mathrm{rms}$ over $1 \mathrm{~s}$. Long-term stability is also assessed, with $380 \mathrm{mrad}$ measured over $1 \mathrm{~h}$. This level of performance is allowed by a hybrid architecture, including a passively CEP-stabilized front-end based on difference frequency generation and an active CEP stabilization loop for the fiber amplifier system, acting on a telecom-grade integrated $\mathrm{LiNbO} 3$ phase modulator. Together with recent demonstrations of temporal compression down to the few-cycle regime, the presented results demonstrate the relevance of the $\mathbf{Y b}$-doped high repetition rate laser for attoscience. () 2019 Optical Society of America
\end{abstract}

https://doi.org/10.1364/OL.44.003909

Carrier-envelope phase (CEP) stabilized few-cycle amplified laser systems are one of the keys enabling attoscience [1,2]. Since the first demonstration of isolated attosecond pulse generation [3], applications requiring a precise control of the electric field have considerably expanded. To date, titanium-doped sapphire (Ti:Sa) amplifier systems have mostly provided the required short and energetic pulses at repetition rates ranging from $50 \mathrm{~Hz}$ to $10 \mathrm{kHz}[4,5]$.

A large number of applications would benefit from further repetition rate scaling to $\geq 100 \mathrm{kHz}$, reducing acquisition times and improving signal-to-noise ratio. This is particularly true for low yield experiments involving coincidence detection [6]. At repetition rates above $10 \mathrm{kHz}$, high-power Ti:Sa amplifiers become complicated and costly, and do not provide the longterm stability and robustness required by today's science. A proposed and demonstrated alternative is using CEP-stable Ti:Sa to seed optical parametric chirped pulse amplifiers (OPCPAs) $[7,8]$. Of particular interest, OPCPAs can be configured to work at different wavelengths opening new opportunities. However, OPCPAs are limited by the low conversion efficiency from the pump to the useable beam $(\sim 15 \%)$ before spatial and temporal distortions occur. Very powerful hence, costly, pump sources have to be employed to generate only a few tens of watts [9]. In the past years, laser sources based on Ytterbium gain material have shown stunning performances with $>$ kilowatt average power and >millijoule energy per pulses $[10,11]$. Furthermore, efficient nonlinear compression of these laser sources down to the few-cycle regime has been demonstrated $[12,13]$ and used to drive high-photon flux extreme ultraviolet (XUV) sources [14,15] through high-harmonic generation (HHG). However, robust CEP stabilization is still missing. This is due to a number of reasons such as noisy fiber oscillator characteristics [16], a large stretching and compression ratio, and enhanced intensity to CEP noise transfer in different nonlinear stages [17]. To date, CEP stabilization of Ytterbium-based amplified systems have been realized in a linear amplification regime at the microjoule level with a regenerative $\mathrm{Yb}: \mathrm{KGW}$ system at $1 \mathrm{MHz}$ seeded by a solid-state $\mathrm{Yb}: \mathrm{KGW}$ oscillator [18] and with a fiber amplifier at $80 \mathrm{MHz}$ seeded by a CEP-stable Ti:Sa oscillator [19]. Both systems revealed CEP fluctuations comparable to Ti:Sa and OPCPA sources, but are not directly scalable in average power for the former one [18] and in pulse energy for the latter one [19]. A CEP-stable Tm-doped linear fiber CPA architecture at $2 \mu \mathrm{m}$ has also been demonstrated recently [20].

In this Letter, we report, to the best of our knowledge, on the first high-energy CEP-stabilized Yb-doped fiber chirped-pulse amplifier (FCPA) system, including a multi-pass cell (MPC) nonlinear compression stage [21]. It delivers $30 \mu \mathrm{J} 96$ fs pulses at $100 \mathrm{kHz}$ and relies on the following several key elements:

- A passively CEP-stable front-end at a central wavelength of $1030 \mathrm{~nm}$.

- A FCPA system, including preamplifiers, a rod-type fiber power amplifier, and a large stretching/compression ratio.

- An active CEP feedback loop, including an in-line infocus f-to-2f interferometer and an integrated electro-optic phase modulator (PM) as an actuator.

The CEP stability is characterized in detail both at the full $100 \mathrm{kHz}$ bandwidth over $1 \mathrm{~s}$ and at $10 \mathrm{kHz}$ over $1 \mathrm{~h}$, revealing 


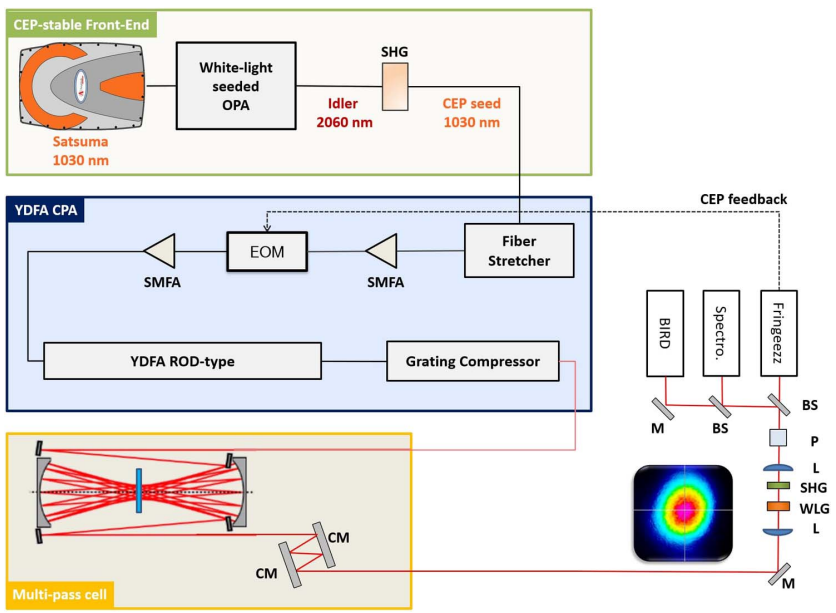

Fig. 1. Experimental setup. SHG, second-harmonic generation; WLG, white light generation; OPA, optical parametric amplification; SMFA, single-mode fiber amplifier; EOM, electro-optical modulator; $\mathrm{CM}$, chirped mirror; $\mathrm{M}$, mirror; L, lens; $\mathrm{P}$, polarizer; $\mathrm{BS}$, beam splitter.

$<400$ mrad CEP stability on a shot-to-shot basis. This is, to the best of our knowledge, the first spectral f-2f CEP measurement ever reported at a repetition rate $>10 \mathrm{kHz}$. Overall, this source demonstrates that $\mathrm{Yb}$-based ultrafast laser technology is compatible with CEP stabilization, paving the way for the future generation of compact and efficient laser drivers for attoscience applications.

The experiment is depicted in Fig. 1. It consists of a passive CEP-stable front-end, seeding a large stretching/compression ratio high-energy FCPA, followed by a MPC temporal compression stage.

The front-end uses a commercially available FCPA (Satsuma, Amplitude Laser) that delivers $40 \mu \mathrm{J} 300 \mathrm{fs}$ pulses at a repetition rate tunable up to $500 \mathrm{kHz}$. After second-harmonic generation (SHG) in a $2 \mathrm{~mm}$ long BBO crystal with $49 \%$ efficiency, this source pumps an optical parametric amplifier (OPA). Both the white light generated signal and OPA pump originate from the same laser pulse train. In this configuration, the idler radiation is inherently CEP-stabilized [22]. Here, the idler wavelength is tuned at $2060 \mathrm{~nm}$ and further converted to $1030 \mathrm{~nm}$ through a second stage of SHG in a $2 \mathrm{~mm}$ long BBO. Up to $400 \mathrm{~nJ}$ of energy per pulse is produced and seeded in the FCPA with a full width at half-maximum pulse duration of $185 \mathrm{fs}$. The repetition rate of the CEP-stable seeder is tunable up to $500 \mathrm{kHz}$ through the control of the OPA pump laser. It is used at $100 \mathrm{kHz}$ throughout this paper. The high-energy FCPA is composed of a chirped fiber Bragg grating stretcher (stretched pulse duration $\sim 350 \mathrm{ps}$ ), followed by two single-mode low-power fiber pre-amplifiers that provide a total gain of $26 \mathrm{~dB}$. Between these amplifiers, a waveguide fiber-coupled $\mathrm{LiNbO}_{3} \mathrm{PM}$ is inserted (insertion loss of $3 \mathrm{~dB}$ ) and used as a CEP control device. The possibility to stabilize the CEP of a Ti:Sa amplified laser using a bulk electro-optic modulator was demonstrated in Ref. [23]. The voltage required to shift the CEP phase by $\pi$ is related to the standard $V_{\pi}$ by

$$
V_{\pi, \mathrm{CEP}}=V_{\pi} \frac{n_{e} r_{33}}{\lambda\left(3 r_{33} \frac{\partial n_{e}}{\partial \lambda}+n_{e} \frac{\partial r_{33}}{\partial \lambda}\right)},
$$
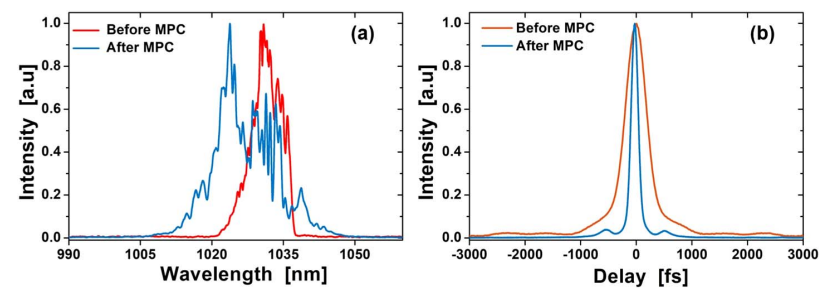

Fig. 2. (a) Spectra and (b) autocorrelation traces at the output of the CPA (red) and MPC-based temporal compression (blue).

where $\lambda$ is the wavelength, $n_{e}$ is the extraordinary refractive index of $\mathrm{LiNbO}_{3}$ and $r_{33}$ is one component of its electro-optic tensor. We estimate the value of the right-hand side fraction in Eq. (1) to be 7 , which is consistent with the experimentally measured value of $V_{\pi \text {,CEP }}$ of $8 \mathrm{~V}$, and the specified $V_{\pi}<2 \mathrm{~V}$. The power amplifier is composed of a $1 \mathrm{~m}$ long rod-type fiber with a mode field diameter of $65 \mu \mathrm{m}$ pumped by a high-power $976 \mathrm{~nm}$ diode laser nominally used at an average power of $10 \mathrm{~W}$. Finally, a highly dispersive $1750 \mathrm{l} / \mathrm{mm} 12 \mathrm{~cm}$ large grating compressor is set up to compress the pulse down to $340 \mathrm{fs}$.

Additional temporal compression is performed by means of a bulk MPC. The MPC is made of two spherical mirrors with a radius of curvature of $200 \mathrm{~mm}$, separated by $240 \mathrm{~mm}$. The pulses recirculate for 15 roundtrips in the MPC in which a $2.3 \mathrm{~mm}$ thick antireflection-coated silica plate is inserted. Here the role of the MPC is twofold.

First, the pulse duration reduction increases the coherence of the white light generation process for CEP measurement. Secondly, it is included as a test bench to study CEP stability in the presence of a highly nonlinear compression stage that must be included in a future high-power source for attosecond physics.

Figure 2 shows the spectral and temporal characteristics of the pulses at the output of the FCPA and after the MPC. At an input energy to the MPC of $30 \mu \mathrm{J}$, limited by the decreasing output temporal pulse quality, the spectrum is broadened from $16 \mathrm{~nm}$ to $45 \mathrm{~nm}$ (measured at $-10 \mathrm{~dB}$ ) leading to a pulse duration of 96 fs out of the MPC (assuming a Gaussian shape for the deconvolution), after chirp removal using $4800 \mathrm{fs}^{2}$ of negative dispersion. The transmission of this stage is $89 \%$.

Finally, an in-line, in-focus $\mathrm{f}$-to- $2 \mathrm{f}$ interferometer setup is used for CEP characterization [24]. A pulse energy of $\sim 1.5 \mu \mathrm{J}$ is sampled out of the MPC beam and focused into a $3 \mathrm{~mm}$ thick YAG crystal for octave-spanning spectral broadening. In the same focus, a $2 \mathrm{~mm}$ thick BBO crystal is used to frequency double the spectral content in the 1100-1150 nm range, generating the spectral interference pattern in the 550 $575 \mathrm{~nm}$ range after a polarizing cube.

We now focus on the in-depth characterization of the CEP stability. Three separate devices are used to detect spectral fringes from the $\mathrm{f}-2 \mathrm{f}$ interferometer, allowing us to investigate different properties.

First, a commercial fast CEP measurement device (Fringeezz, Fastlite) is used at the output of the f-to-2f interferometer to acquire single-shot CEP values at a $10 \mathrm{kHz}$ sampling rate. This signal is fed to a digital proportionalintegral-derivative (PID) servo-controller that drives the PM. Figure 3(a) shows the in-loop CEP power spectral density (PSD) and RMS integrated phase noise (IPN) in open and closed loop operation, acquired over $10 \mathrm{~s}$. In the closed loop case, an 

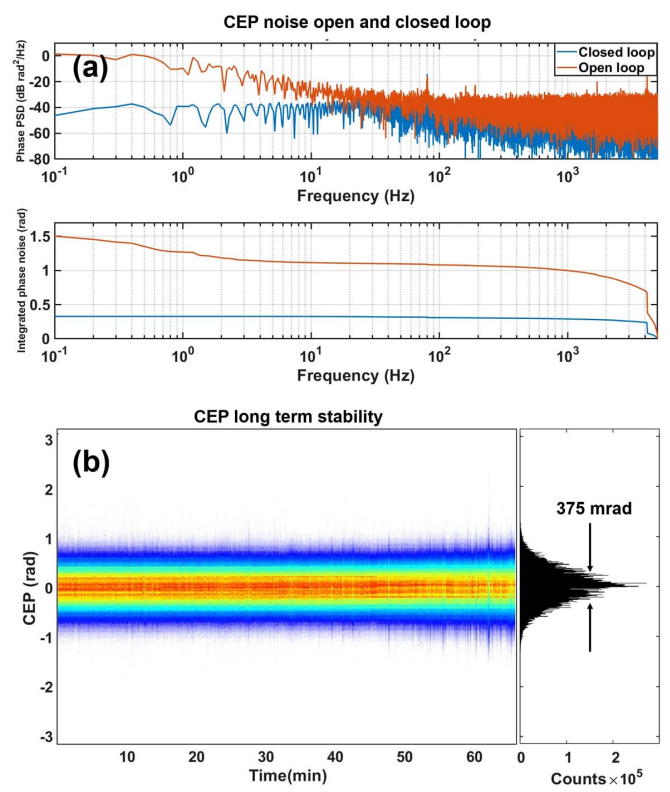

Fig. 3. In-loop single-shot CEP characterization at a $10 \mathrm{kHz}$ sampling rate (a) CEP PSD and IPN for open and closed loop operations. (b) Left: the CEP density plot in closed loop over $60 \mathrm{~min}$. Right: corresponding CEP histogram.

overall IPN of $320 \mathrm{mrad}$ is obtained, owing to the strong reduction of low-frequency noise allowed by the feedback loop. A long-term CEP density plot (one histogram per second) over $1 \mathrm{~h}$ is also shown in Fig. 3(b) and yields an overall IPN of $375 \mathrm{mrad}$, demonstrating the robustness of the system.

Secondly, while using the first device to stabilize the CEP out-of-loop characterization is performed by measuring the spectral fringes using a fast spectrometer. The minimum integration time of $30 \mu \mathrm{s}$ allows us to measure CEP fluctuations integrating over three pulses, at a maximum sampling rate of $330 \mathrm{~Hz}$. Figure 4(a) shows the fringe spectrum acquired on the first acquisition (used to measure the fringe visibility), Fig. 4(b) shows the fringe pattern as a function of time, Fig. 4(c) shows the corresponding CEP variation as a function of time [25], and Fig. 4(d) shows the overall CEP histogram over $3 \mathrm{~s}$ for three different integration times of $30 \mu \mathrm{s}, 100 \mu \mathrm{s}$, and $1 \mathrm{~ms}$, corresponding, respectively, to 3, 10, and 100 shots averaging. The IPN increases from $56 \mathrm{mrad}$ to $115 \mathrm{mrad}$ and $214 \mathrm{mrad}$ for decreasing integration times, with corresponding noise bandwidths of $[0.3 \mathrm{~Hz}-1 \mathrm{kHz}],[0.3 \mathrm{~Hz}-10 \mathrm{kHz}]$, and $[0.3 \mathrm{~Hz}-33 \mathrm{kHz}]$. An interesting point is to compare the measured fringe contrast, which is sometimes used as a way to characterize the CEP stability [26]. In our case, it remains essentially constant, with a visibility increasing from $68.3 \%-68.9 \%$ from the less to the most integrated case. Therefore, this visibility is not a meaningful indication of CEP stability in these conditions.

Finally, a third CEP measurement is performed out-of-loop, with a focus on measuring the shot-to-shot CEP at $100 \mathrm{kHz}$ using the Beat Interferometer for Rapid Detection (BIRD, Amplitude Laser) technique [27]. This measurement is completely analog and based on the use of the difference signal from two photomultipliers detecting the signals of a spatially dispersed spectral fringe hitting the apex of a prism. Figure 5(a) shows the closed loop PSD and corresponding IPN on the frequency range $[1 \mathrm{~Hz}-50 \mathrm{kHz}$. The total integrated RMS CEP noise is $325 \mathrm{mrad}$ for an acquisition time of $1 \mathrm{~s}$. This measurement confirms the Fringeezz measurement, but over the full bandwidth. To the best of our knowledge, this is the first time that a spectral $\mathrm{f}$-to- $2 \mathrm{f}$ measurement has been performed at $100 \mathrm{kHz}$. The only other report of shot-to-shot CEP measurement at $100 \mathrm{kHz}$ that we are aware of is based on stereo above threshold ionization, as reported in Ref. [28].

Lastly, we report on intensity fluctuations of the complete laser source, which are known to be correlated with CEP noise through various amplitude-to-phase noise transfer mechanisms $[29,30]$. As an example, self-phase modulation in the white light generation (inside the OPA and the f-to-2f), amplification stages, and MPC could cause such a transfer. We characterize
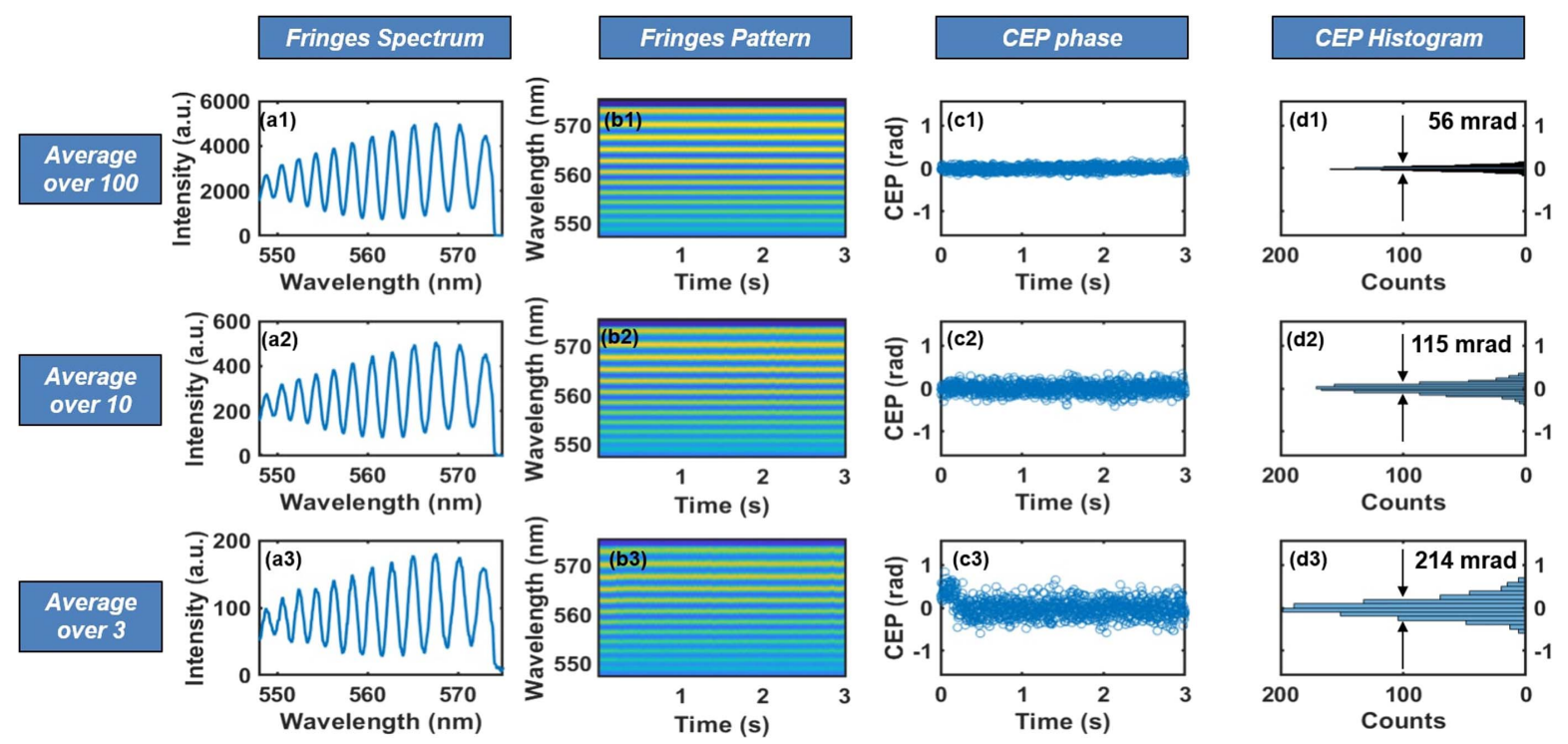

Fig. 4. Out-of-loop f-2f spectral fringe measurement with a spectrometer. (a1)-(a3) Single acquisition of spectral fringes with integration times corresponding to 100, 10, and 3 pulses, respectively. (b1)-(b3) Corresponding fringe pattern evolution as a function of time over 3 s. (c1)-(c3) Corresponding CEP drift as a function of time. (d1)-(d3) Histograms of CEP fluctuations. 

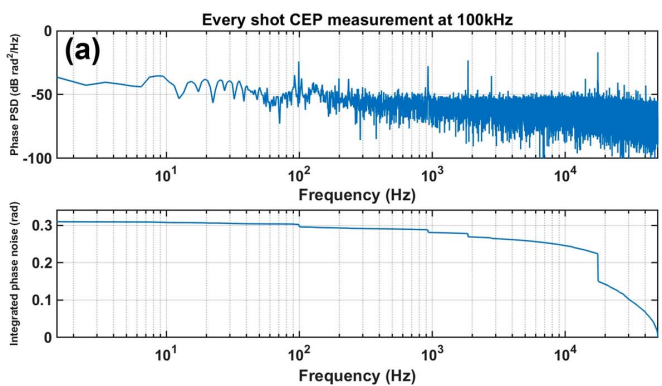

Frequency $(\mathrm{Hz})$
RIN output MPC
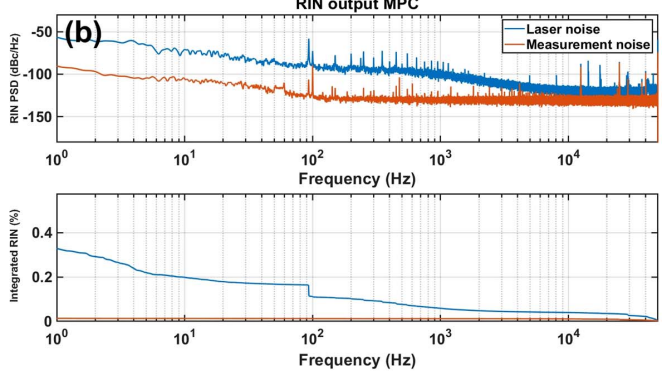

Fig. 5. (a) Full PSD and IPN from a single-shot, every-shot measurement. (b) PSD and IRIN at the output of the laser source.

our system in terms of relative intensity noise. The measurement setup consists of a Si photodiode, a low-pass filter at Nyquist frequency, and an oscilloscope. The RIN PSD and integrated RMS RIN (IRIN) measured after the MPC are shown in Fig. 5(b). The total IRIN is $0.32 \%$ in the $[1 \mathrm{~Hz}-50 \mathrm{kHz}$ ] bandwidth. For reference, the IRIN of the pump laser of the OPA measured in the same conditions is $0.5 \%$, indicating that saturation in the amplifiers throughout the system reduces the IRIN. We also point out a RIN spectral peak at $100 \mathrm{~Hz}$ which is clearly correlated to a peak at the same frequency in the CEP PSD [see Fig. 5(a)]. This is clear evidence of an amplitude-tophase noise transfer. As expected, therefore, a low value of IRIN is paramount in the design of such a CEP-stable system.

In conclusion, we demonstrated the CEP stabilization of an ultrafast FCPA followed by an MPC-based nonlinear temporal compression stage. This proof-of-principle system delivers $30 \mu \mathrm{J} 96 \mathrm{fs}$ pulses centered at $1030 \mathrm{~nm}$, at a $100 \mathrm{kHz}$ repetition rate with a full-bandwidth single-shot CEP stability better than $400 \mathrm{mrad}$ and RMS IRIN of $0.32 \%$. These results bring clear evidence of the compatibility of all subsystems, including stretcher/compressor units, single-mode fiber amplifiers, large-mode-area power amplifiers, and nonlinear compression in an MPC, with CEP stabilization. Taking into account the already demonstrated high-energy, high-power [11] amplifier architectures, as well as the high-efficiency nonlinear compression schemes [31], the system performances could realistically be scaled. We believe that this Letter paves the way to the development of compact, robust, CEP-stable, high-power $(>100 \mathrm{~W})$, high-energy $(>500 \mu \mathrm{J})$, few-cycle sources particularly attractive for a wide range of applications such as XUV generation through HHG, attoscience, coincidence spectroscopy, and nanoscale imaging.

Funding. Conseil Départemental de l'Essonne (ASTRE Sophie); FP7 People: Marie-Curie Actions (PEOPLE) (H2020MSCA-ITN-2014-641789-MEDEA); Agence Nationale de la Recherche (ANR) (ANR-10-LABX-0039-PALM).

\section{REFERENCES}

1. F. Krausz and M. Ivanov, Rev. Mod. Phys. 81, 163 (2009).

2. G. Sansone, L. Poletto, and M. Nisoli, Nat. Photonics 5, 655 (2011).

3. G. Sansone, E. Benedetti, F. Calegari, C. Vozzi, L. Avaldi, R. Flammini, L. Poletto, P. Villoresi, C. Altucci, R. Velotta, S. Stagira, S. De Silvestri, and M. Nisoli, Science 314, 443 (2006).

4. G. Gademann, F. Plé, P.-M. Paul, and M. J. Vrakking, Opt. Express 19, 24922 (2011).

5. J. Klein and J. D. Kafka, Nat. Photonics 4, 289 (2010).

6. M. Weger, J. Maurer, A. Ludwig, L. Gallmann, and U. Keller, Opt. Express 21, 21981 (2013).

7. J. Rothhardt, S. Demmler, S. Hädrich, J. Limpert, and A. Tünnermann, Opt. Express 20, 10870 (2012).

8. O. Chalus, P. K. Bates, M. Smolarski, and J. Biegert, Opt. Express 17, 3587 (2009).

9. R. Budriūnas, T. Stanislauskas, J. Adamonis, A. Aleknavičius, G. Veitas, D. Gadonas, S. Balickas, A. Michailovas, and A. Varanavičius, Opt. Express 25, 5797 (2017).

10. M. Müller, M. Kienel, A. Klenke, T. Gottschall, E. Shestaev, M. Plötner, J. Limpert, and A. Tünnermann, Opt. Lett. 41, 3439 (2016).

11. B. Dannecker, J.-P. Negel, A. Loescher, P. Oldorf, S. Reichel, R. Peters, T. Graf, and M. A. Ahmed, Opt. Commun. 429, 180 (2018).

12. L. Lavenu, M. Natile, F. Guichard, Y. Zaouter, M. Hanna, E. Mottay, and P. Georges, Opt. Express 25, 7530 (2017).

13. J. Rothhardt, S. Hädrich, A. Klenke, S. Demmler, A. Hoffmann, T. Gotschall, T. Eidam, M. Krebs, J. Limpert, and A. Tünnermann, Opt. Lett. 39, 5224 (2014).

14. S. Hädrich, J. Rothhardt, M. Krebs, S. Demmler, A. Klenke, A. Tünnermann, and J. Limpert, J. Phys. B 49, 172002 (2016).

15. A. I. Gonzalez, G. Jargot, P. Rigaud, L. Lavenu, F. Guichard, A. Comby, T. Auguste, O. Sublemontier, M. Bougeard, Y. Zaouter, P. Georges, M. Hanna, and T. Ruchon, J. Opt. Soc. Am. B 35, A6 (2018).

16. N. Raabe, T. Feng, M. Mero, H. Tian, Y. Song, W. Hänsel, R. Holzwarth, A. Sell, A. Zach, and G. Steinmeyer, Opt. Lett. 42, 1068 (2017)

17. F. Lücking, A. Trabattoni, S. Anumula, G. Sansone, F. Calegari, M. Nisoli, T. Oksenhendler, and G. Tempea, Opt. Lett. 39, 2302 (2014).

18. T. Balčiūnas, T. Flöry, A. Baltuška, T. Stanislauskas, R. Antipenkov, A. Varanavičius, and G. Steinmeyer, Opt. Lett. 39, 1669 (2014).

19. T. Saule, S. Holzberger, O. De Vries, M. Plötner, J. Limpert, A. Tünnermann, and I. Pupeza, Appl. Phys. B 123, 17 (2017).

20. C. Gaida, T. Heuermann, M. Gebhardt, E. Shestaev, T. P. Butler, D. Gerz, N. Lilienfein, P. Sulzer, M. Fischer, R. Holzwarth, and A. Leitenstorfer, Opt. Lett. 43, 5178 (2018).

21. M. Hanna, X. Délen, L. Lavenu, F. Guichard, Y. Zaouter, F. Druon, and P. Georges, J. Opt. Soc. Am. B 34, 1340 (2017).

22. G. Cerullo, A. Baltuška, O. D. Mücke, and C. Vozzi, Laser Photonics Rev. 5, 323 (2011).

23. O. Gobert, P. Paul, J. Hergott, O. Tcherbakoff, F. Lepetit, P. D'Oliveira, F. Viala, and M. Comte, Opt. Express 19, 5410 (2011).

24. M. Kakehata, H. Takada, Y. Kobayashi, K. Torizuka, Y. Fujihira, T. Homma, and H. Takahashi, Opt. Lett. 26, 1436 (2001).

25. M. Takeda, H. Ina, and S. Kobayashi, J. Opt. Soc. Am. 72, 156 (1982).

26. C.-H. Lu, T. Witting, A. Husakou, M. J. Vrakking, A. Kung, and F. J. Furch, Opt. Express 26, 8941 (2018).

27. S. Koke, C. Grebing, B. Manschwetus, and G. Steinmeyer, Opt. Lett. 33, 2545 (2008).

28. D. Hoff, F. J. Furch, T. Witting, K. Rühle, D. Adolph, A. M. Sayler, M. J. Vrakking, G. G. Paulus, and C. P. Schulz, Opt. Lett. 43, 3850 (2018)

29. A. Baltuska, M. Uiberacker, E. Goulielmakis, R. Kienberger, V. S. Yakovlev, T. Udem, T. W. Hansch, and F. Krausz, IEEE J. Sel. Top. Quantum Electron. 9, 972 (2003).

30. C. Li, E. Moon, H. Wang, H. Mashiko, C. M. Nakamura, J. Tackett, and Z. Chang, Opt. Lett. 32, 796 (2007).

31. L. Lavenu, M. Natile, F. Guichard, X. Délen, M. Hanna, Y. Zaouter, and P. Georges, Opt. Express 27, 1958 (2019). 